\title{
Prognostic staging of acute pulmonary embolism: are we closer to the holy grail?
}

\author{
Adam Torbicki
}

Affiliations: Dept of Pulmonary Circulation and Thromboebolic Diseases, Centre of Postgraduate Medical Education, ECZ-Otwock, Otwock, Poland.

Correspondence: Adam Torbicki, Dept of Pulmonary Circulation and Thromboebolic Diseases, Center of Postgraduate Medical Education, ECZ-Otwock, ul. Borowa 14/18, 05-400 Otwock, Poland.

E-mail: adam.torbickidecz-otwock.pl

0

@ERSpublications

Tachycardia, borderline systolic pressure, RV dysfunction and injury: new definition for intermediate-risk PE? http://ow.ly/wJu2d

\begin{abstract}
Management strategy and its influence on the outcomes for patients with acute pulmonary embolism (PE) has been an area of chronic debate and unresolved controversies. During the last decade at least 50 publications have provided important evidence on prognostic markers, with the hope of improving risk stratification and, consequently, treatment decisions for PE. Such markers ranged from simple clinical signs to variables, which require sophisticated laboratory tests and modern cardiovascular imaging. Paradoxically, messages most relevant to a practicing clinician confronting a patient with acute PE, so far, seem to emerge from clinical evaluation. Signs of shock or significant hypotension became universally accepted markers of high risk PE, justifying emergency thrombolysis [1]. More recently, several clinical scores have been reliably validated to identify patients at a very low risk of adverse outcomes, despite confirmed PE [2-4]. If adequately anticoagulated, such patients may be offered an option of early discharge and ambulatory therapy [5].
\end{abstract}

The trial by Bova et al. [6] attempts to solve a remaining important clinical problem, i.e. that of identifying patients with acute $\mathrm{PE}$ who are at a particularly high risk of adverse outcome, despite normal systemic arterial pressure. Previous similar attempts have not been successful, mostly because of a low positive prognostic value of individual laboratory-risk markers for early PE-related mortality, in this particular patient subgroup.

Nevertheless, in initially hemodynamically stable patients, deaths do occur either because of early recurrence of PE or the decompensation of the right ventricle that has been submitted to excessive and prolonged overload. Progressive dilatation of right heart chambers, functional tricuspid regurgitation, increasing wall strain and oxygen myocardial, in a setting of diminished functional right ventricular coronary perfusion and hypoxaemia, may initiate and feed a rapid vicious circle, which leads to the haemodynamic collapse in an initially normotensive patient [1]. Can we identify those patients before it occurs?

While pathophysiologically sound and correlated with outcome, the imaging or biochemical markers of right ventricular dysfunction were, so far, only able to identify patients with PE facing an uncomplicated clinical course. This was also true for laboratory markers for myocardial injury. Several trials suggested that joint consideration of two different markers may provide additive positive predictive power if both were found positive, particularly when they had different pathophysiological significance. As an example, a positive troponin test, suggesting myocardial injury in the presence of echocardiographic signs of right ventricular dysfunction was reported to be related to $38 \%$ mortality within 30 days [7]. Similarly, a biomarker-based approach suggested a 40-day PE-related mortality of 33\% in patients with both elevated plasma N-terminal pro-brain natriuretic peptide (a humoral marker for myocardial stretch) and a positive troponin test [8].

Received: April 172014 | Accepted: April 232014

Conflict of interest: Disclosures can be found alongside the online version of this article at erj.ersjournals.com

Copyright @ERS 2014 
Based on those and other reports a risk stratification scheme, which considered an incremental prognostic value of right ventricular dysfunction and myocardial injury was suggested by the European Society of Cardiology (ESC) guidelines on acute PE [1]. It has been estimated that early mortality in normotensive patients with positive laboratory risk markers may reach up to $15 \%$. However, no validated criteria could be provided, as cut-off values suggested for individual prognostic markers varied between the published trials. A recent study, which included patients from the Italian Pulmonary Embolism Registry (IPER), confirmed a prognostic gradient between normotensive PE patients with no, single or both positive-risk markers for right ventricular dysfunction and myocardial injury [9]. However, the real-life PE mortality was found to be much lower than previously reported by individual trials, which assessed the prognostic power of various biomarkers and their combinations.

The Pulmonary Embolism Thrombolysis Study (PEITHO), a recent multicentre, randomised, controlled trial, assessed the rationale for thrombolysis in intermediate-risk patients defined as normotensive, but with a positive troponin test and signs of right ventricular dysfunction on cardiovascular imaging [10]. Similarly to the study involving IPER patients [9], the PEITHO trial found that early mortality (up to 7 days) among patients initially treated with heparin alone was low (1.8\%) [11]. Primary thrombolysis drastically reduced the frequency of secondary haemodynamic decompensation and the need for rescue thrombolysis. However, it failed to provide a survival benefit (mortality at day 7 was $1.2 \%$ ), which could justify markedly increased incidence of major bleeding, intracranial haemorrhage and stroke.

PEITHO has left us with several unanswered questions. 1) Should we abandon attempts to further riskstratify normotensive PE patients in search of those requiring more aggressive initial treatment, as there is no such subgroup? 2) Should we continue this search but use more stringent criteria than the PEITHO trial, which allowed enrolment of patients with relatively mild signs of right ventricular dysfunction on echocardiography? 3) Should we reconsider and extend the panel of markers necessary to identify patients in need of early aggressive, but more risky treatments, despite normal systemic blood pressure?

The trial by Bova et al. [6] supports the third concept by suggesting a new, more comprehensive, panel of clinical and laboratory markers that result in a more powerful prognostic score. Compared to previous trials [12-14] the authors may be more successful because of a much greater population from which the prognostic data could be derived. Bova et al. [6] managed to access and jointly analyse the databases of six large recent trials, which prospectively collected data on baseline clinical presentation and results of laboratory tests, as well as on 30-day outcomes of almost 3000 normotensive patients with PE [15-20]. The trial was not free from limitations; nonhomogeneous criteria defining a positive troponin test, as well as different echocardiographic criteria of right ventricular overload across the merged trials, could not be avoided. In addition not all trials could offer a complete panel of potentially relevant prognostic markers. Even so, the reported results appear clinically appealing. The main message from the trial is that we should not restrict our prognostic evaluation to laboratory markers or to clinical markers alone, but consider them jointly. While apparently obvious, this message in fact contradicts the two currently used strategies of staging the severity of normotensive PE. Those strategies either focused on evaluation of clinical prognostic score, such as the pulmonary embolism severity index, or on sophisticated markers of right ventricle overload and injury, but somehow failed to integrate the potentially available information. This situation was partly due to different goals driving the two strategies: the identification of low-risk patients suitable for early home treatment [21]; and the identification of patients requiring aggressive management strategy [1]. Indeed the ESC strategy of prognostic staging of patients with PE who potentially needed early aggressive therapy after excluding shock or hypotension, required no further clinical assessment. The trial of Bova et al. [6] suggests that it may be useful to also take into account borderline values of systemic blood pressure as well as the presence of tachycardia. In other words, they remind us that the clinical presentation is indeed a continuum and that simple clinical data suggesting that we are dealing with a potentially unstable patient should not be neglected. When considered together with the results of testing for laboratory risk markers this seem to add additional power to prognostic stratification. It was interesting to see that with this approach Bova et al. [6] would be able to identify a subgroup of normotensive PE patients with a 30-day PE-related mortality of 15\%, as expected by the ESC guidelines published in 2008 [1].

In practical terms this would mean that after excluding shock and hypotension we should also take note of patients with systolic blood pressures (SBP) in the range of 90-100 $\mathrm{mmHg}$ (2 score points) and with tachycardia and heart rate $(\mathrm{HR}) \geqslant 100 \mathrm{bpm}$ (1 score point). These are in fact two components of a decomposed so called "shock index" defined as HR/SBP [22]. Positive troponin (2 points) and right ventricular dysfunction (2 points) complete the panel of independent prognostic risk markers. Of note, patients enrolled in the PEITHO trial, based on increased troponin levels and right ventricular dysfunction, would still need either an SBP 90-100 mmHg or a tachycardia of $\geqslant 110 \mathrm{bpm}$ (or both) to be considered at intermediate risk of early death due to PE according to the criteria suggested by the current trial (Bova score more than four). 
Despite suggestive results, Bova et al. [6] were cautious in their management recommendations and, even in patients qualified to the highest risk group, advocated close monitoring rather than early aggressive therapy. In fact more straightforward statements would require not only an external validation of the performance of suggested prognostic score but also a new prospective randomised therapeutic trial. It could be similar to PEITHO trial, but with an extended and more demanding set of prognostic markers qualifying for early aggressive therapy. In view of 12 years, which were needed to accomplish the PEITHO project and publish its results, we may need patience. In the meantime standardised cut-off values for biochemical markers should be implemented and a consensus on most suitable imaging markers of right ventricular dysfunction should be reached $[23,24]$. Only then can prospective research approach the problem of identification of normotensive patients with PE at risk of death, elevated to a level justifying early aggressive therapy unloading the right ventricle. Bova et al. [6] have, with their trial, brought us one step closer to this holy grail.

\section{References}

1 Torbicki A, Perrier A, Konstantinides S, et al. Guidelines on the diagnosis and management of acute pulmonary embolism: the Task Force for the Diagnosis and Management of Acute Pulmonary Embolism of the European Society of Cardiology (ESC). Eur Heart J 2008; 29: 2276-2315.

2 Aujesky D, Obrosky DS, Stone RA, et al. A prediction rule to identify low-risk patients with pulmonary embolism. Arch Intern Med 2006; 166: 169-175.

3 Aujesky D, Perrier A, Roy PM, et al. Validation of a clinical prognostic model to identify low-risk patients with pulmonary embolism. J Intern Med 2007; 261: 597-604.

4 Jiménez D, Aujesky D, Moores L, et al. Simplification of the pulmonary embolism severity index for prognostication in patients with acute symptomatic pulmonary embolism. Arch Intern Med 2010; 170: 1383-1389.

5 Sam A, Sánchez D, Gómez V, et al. The shock index and the simplified PESI for identification of low-risk patients with acute pulmonary embolism. Eur Respir J 2011; 37: 762-766.

6 Bova C, Sanchez O, Prandoni P, et al. Identification of intermediate-risk patients with acute symptomatic pulmonary embolism. Eur Respir J 2014; 44: 694-703.

7 Scridon T, Scridon C, Skali H, et al. Prognostic significance of troponin elevation and right ventricular enlargement in acute pulmonary embolism. Am J Cardiol 2005; 96: 303-305.

8 Kostrubiec M, Pruszczyk P, Bochowicz A, et al. Biomarker-based risk assessment model in acute pulmonary embolism. Eur Heart J 2005; 26: 2166-2172.

9 Becattini C, Casazza F, Forgione C, et al. Acute pulmonary embolism: external validation of an integrated risk stratification model. Chest 2013; 144: 1539-1545.

10 Meyer G, Vicaut E, Danays T, et al. Fibrinolysis for patients with intermediate-risk pulmonary embolism. N Engl J Med 2014; 370: 1402-1411.

11 Meyer G, Vicaut E, Danays T, et al. Fibrinolysis for patients with intermediate-risk pulmonary embolism. $N$ Engl J Med 2014; 370: 1402-1411.

12 Jiménez D, Kopecna D, Tapson V, et al. Derivation and validation of multimarker prognostication for normotensive patients with acute symptomatic pulmonary embolism. Am J Respir Crit Care Med 2014; 189: 718-726.

13 Dellas C, Tschepe M, Seeber V, et al. A novel H-FABP assay and a fast prognostic score for risk assessment of normotensive pulmonary embolism. Thromb Haemost 2014; 111: 996-1003.

14 Sanchez O, Trinquart L, Planquette B, et al. Echocardiography and pulmonary embolism severity index have independent prognostic roles in pulmonary embolism. Eur Respir J 2013; 42: 681-688.

15 Bova C, Pesavento R, Marchiori A, et al. Risk stratification and outcomes in hemodynamically stable patients with acute pulmonary embolism: a prospective, multicentre, cohort study with three months of follow-up. J Thromb Haemost 2009; 7: 938-944.

16 Sanchez O, Trinquart L, Caille V, et al. Prognostic factors for pulmonary embolism: the prep study, a prospective multicenter cohort study. Am J Respir Crit Care Med 2010; 181: 168-173.

17 Jiménez D, Aujesky D, Moores L, et al. Combinations of prognostic tools for identification of high-risk normotensive patients with acute symptomatic pulmonary embolism. Thorax 2011; 66: 75-81.

18 Vanni S, Nazerian P, Pepe G, et al. Comparison of two prognostic models for acute pulmonary embolism: clinical vs. right ventricular dysfunction-guided approach. J Thromb Haemost 2011; 9: 1916-1923.

19 Lankeit M, Jiménez D, Kostrubiec M, et al. Predictive value of the high-sensitivity troponin $\mathrm{T}$ assay and the simplified pulmonary embolism severity index in hemodynamically stable patients with acute pulmonary embolism: a prospective validation study. Circulation 2011; 124: 2716-2724.

20 Jiménez D, Lobo JL, Monreal M, et al. Prognostic significance of multidetector CT in normotensive patients with pulmonary embolism: results of the protect study. Thorax 2014; 69: 109-115.

21 Aujesky D, Roy PM, Verschuren F, et al. Outpatient versus inpatient treatment for patients with acute pulmonary embolism: an international, open-label, randomised, non-inferiority trial. Lancet 2011; 378: 41-48.

22 Toosi MS, Merlino JD, Leeper KV. Prognostic value of the shock index along with transthoracic echocardiography in risk stratification of patients with acute pulmonary embolism. Am J Cardiol 2008; 101: 700-705.

23 Henzler T, Roeger S, Meyer M, et al. Pulmonary embolism: CT signs and cardiac biomarkers for predicting right ventricular dysfunction. Eur Respir J 2012; 39: 919-926.

24 Pruszczyk P, Goliszek S, Lichodziejewska B, et al. Prognostic value of echocardiography in normotensive patients with acute pulmonary embolism. JACC Cardiovasc Imaging 2014; 7: 553-560. 